\title{
Recent results from Daya Bay experiment
}

\author{
Dmitry V.Naumov ${ }^{1, a}$, on behalf of the Daya Bay Collaboration \\ ${ }^{1}$ Joliot-Curie, 6, Dubna, Russia, 141980
}

\begin{abstract}
This manuscript is a short summary of my talk given at ICNFP2014 Conference. Here we report on new results of $\sin ^{2} 2 \theta_{13}$ and $\Delta m_{\mathrm{ee}}^{2}$ measurements, search for the sterile neutrino within $10^{-3} \mathrm{eV}^{2}<\Delta m_{41}^{2}<0.1 \mathrm{eV}^{2}$ domain and precise measurement of the reactor absolute antineutrino flux.
\end{abstract}

\section{Introduction}

Generations of leptons (and similarly quarks) mix in their interactions with $W^{ \pm}$bosons within the Standard Model. The mixing is governed by a unitary matrix of dimension equal to the numer of lepton families. For three lepton's generations assuming the Dirac nature of neutrino the corresponding $3 \times 3$ Pontecorvo-Maki-Nakagawa-Sakata (PMNS) matrix is conviniently described by three mixing angles $\theta_{12}, \theta_{23}$ and $\theta_{13}$ and one CP-violating phase $\delta$. Two more CP-violating phases are required to describe the Majorana neutrinos.

The mixing in the lepton sector leads to a spectacular phenomenon of flavour transformation over time and space - the so called neutrino oscillations first described by Pontecorvo [1,2]. Nowdays the theory of neutrino oscillations is rather well established in both relativistic quantum mechanics and within the framework of quantum field theory [3-13].

Experimentally neutrino oscillations is also a well established phenomenon. Two mixing angles $\theta_{12}$ and $\theta_{23}$ are accurately measured by solar, reactor, atmospheric and accelerator neutrino experiments [14-16] assuming neutrino oscillation hypothesis as an explanation of observed rates of appearance and disappearance of neutrino flavours. Under the same hypothesis two mass squared differences are measured as well. First is $\Delta m_{21}^{2}$ and second is $\Delta m_{\mu \mu}^{2}$ which is a flavour mixture of $\Delta m_{31}^{2}$ and $\Delta m_{32}^{2}$ since current experiments have little sensitivity to the mass hierarchy. Atmospheric and accelerator neutrino experiments measure $\Delta m_{\mu \mu}^{2} \simeq \sin ^{2} \theta_{12} \Delta m_{31}^{2}+\cos ^{2} \theta_{12} \Delta m_{32}^{2}+$ $2 \Delta m_{21}^{2} \sin \theta_{12} \cos \theta_{12} \sin \theta_{13} \tan \theta_{23} \cos \delta$ [17]. Till 2012 the value of $\theta_{13}$ was unknown. A general feeling was that this angle could be very small as Chooz experiment provided an upper limit $\sin ^{2} 2 \theta_{13}<0.15$ [18]. This angle could be measured by both accelerator and reactor neutrinos and a number of experiments (MINOS, T2K, Double Chooz, RENO and Daya Bay) began a race for it. First indications for non zero value of $\theta_{13}$ came from MINOS [19], T2K [20] and Double Chooz [21] in 2011. Also the global analysis of solar and KamLAND data indicated for non-zero value of $\theta_{13}$ [22]. However none of these indications reached a significance even of three standard deviations.

The discovery of non zero value of $\theta_{13}$ was done by a reactor experiment Daya Bay which observed a deficit of $\bar{v}_{e}$ flux at the far site $R=0.940 \pm 0.011$ (stat) \pm 0.004 (syst) which can be explained due

\footnotetext{
ae-mail: dnaumov@jinr.ru
} 
to neutrino oscillations with $\sin ^{2} 2 \theta_{13}=0.092 \pm 0.016$ (stat) \pm 0.005 (syst) [23] in a three-neutrino framework. Soon after RENO Collaboration confirmed this result [24]. A solid determination of a relatively large value of $\theta_{13} \simeq 9^{\circ}$ opened possibilities to study the neutrino mass hierarchy and $\delta$.

In what follows the most recent results of the Daya Bay Collaboration are reviewed. For the yet unpublished results please refer [25, 26]. In Sec. 2 we briefly review the Daya Bay detector and energy model. Please refer the following papers [27, 28] for the event selection. In Sec. 3 we report on new Daya Bay results of neutrino oscillations, sterile neutrino search, measurement of reactor antineutrino flux. Finally, in Sec. 4 we draw our conclusions.

\section{Day Bay experiment}

The Daya Bay experiment is described in details in $[29,30]$. Here we will only briefly recall the main points. There are three experimental halls (EHs) - two "near" and one "far" which contain functionally identical, antineutrino detectors (ADs) surrounded by a pool of ultra-pure water segmented into two regions, the inner water shield and outer water shield, which are instrumented with photomultiplier tubes (PMTs). The Daya Bay experiment uses three-zones antineutrino detectors (AD) schematically shown in the left panel of Fig. 1.
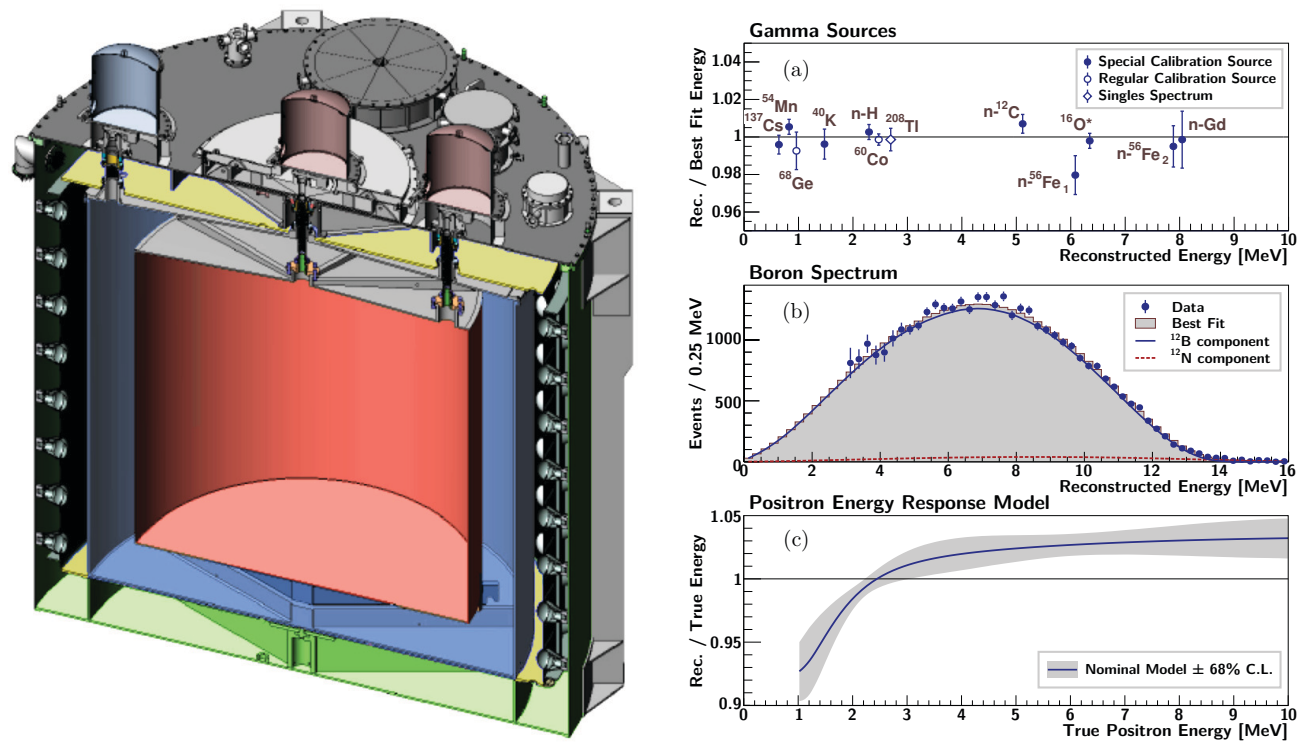

Figure 1. Left panel: three zones Daya Bay antineutrino detector. Right panel:(a) Ratio of the reconstructed to best-fit energies of $\gamma$ lines from calibration sources and singles spectra. The total uncertainty on each ratio is shown as the error bars. (b) Reconstructed energy spectrum (points) compared to the sum (shaded area) of the ${ }^{12} \mathrm{~B}$ (solid line) and ${ }^{12} \mathrm{~N}$ (dashed line) components of the best-fit energy response model. The error bars represent the statistical uncertainties. (c) AD energy response model for positrons.

The inner zone is filled by 20 tons of gadolinium (Gd) doped liquid scintillator (LS) contained in acrylic vessel. The middle zone is filled by 20 tons of LS without gadolinium contained in acrylic vessel. The outer zone is filled by 40 tons of mineral oil. Both inner and middle zones are used to detect $\bar{v}_{e}$ via inverse beta decay (IBD) reaction $\bar{v}_{e}+p \rightarrow e^{+}+n$. The IBD identification exploites the 
time structure of the IBD event - a prompt signal due to $e^{+}$energy loss and subsequent annihilation with $e^{-}$is followed by a recoil neutron capture. The neutron can be captured by Gd nucleus or by proton. We call the corresponding analyses as "Gd analysis" and "nH analysis" respectively. Apparently, the inner zone of $\mathrm{AD}$ is the only target of $\bar{v}_{e}$ for the $\mathrm{nGd}$ analysis while both inner and middle zones serve as the targets for the $\mathrm{nH}$ analysis. The outer zone is used to suppress the background and external radioactivity from PMT and stainless steel structures. Also it suppresses the scintillation in the outer region. The inside volume is viewed by 192 8-inch Hamamatsu PMTs. On average $1 \mathrm{MeV}$ of released energy inside of LS corresponds to about 163 photoelectrons detected by PMTs. The energy resolution is estimated as $\left(7.5 / \sqrt{E_{\mathrm{vis}} / \mathrm{MeV}}+0.9\right) \%$. The ADs are a subject of systematic calibrations compaigns regularly checking the energy response of ADs.

Interpretation of the observed prompt energy spectra requires mapping of the detector response to $e^{+}, e^{-}$and $\gamma$ with the true released visible energy $\left(E_{\text {true }}\right)$ to the reconstructed energy $\left(E_{\text {rec }}\right) . E_{\text {rec }}$ is determined by scaling the measured total charge with a position-dependent correction [29, 31]. Right panel of Fig. 1 compares the best-fit energy model with the single-gamma, multi-gamma and continuous ${ }^{12} \mathrm{~B}$ data used to determine the model parameters. As additional validation, the energy model prediction for the continuous $\beta+\gamma$ spectra from ${ }^{212} \mathrm{Bi},{ }^{214} \mathrm{Bi}$ and ${ }^{208} \mathrm{Tl}$ decays was compared with the data and found to be consistent.

\section{Results}

\subsection{Neutrino oscillation analyses}

Two more oscillations analyses have been carried out since publications [23, 31]. First is nGd analysis [27] and the second is $\mathrm{nH}$ analysis with largely independent systematics and event selection [28]. Let us begin with a discussion of the results of the first analysis. The rate uncertainty of the background is slightly reduced compared to the previous analyses [23, 31] due to the increased statistics. The analysis includes energy shape information by applying the energy nonlinearity correction shown in the right panel of Fig. 1 to the positron spectrum and measuring the energy shape distribution of the five background sources. The spectral uncertainties of the five backgrounds are included as uncorrelated among energy bins in the $\chi^{2}$ fit of the oscillation parameters, to allow all possible spectral models consistent with the data. The combined rate and spectral analysis yields $\sin ^{2} 2 \theta_{13}=0.084 \pm 0.008$ and $\left|\Delta m_{e e}^{2}\right|=\left(2.44_{-0.11}^{+0.10}\right) \times 10^{-3} \mathrm{eV}^{2}$ with $\chi^{2} / \mathrm{NDF}=134.7 / 146$. The corresponding measured prompt energy spectrum is compared to the expectations assuming no oscillations and oscillations with best fit parameters as measured by Daya Bay is shown in the left panel of Fig. 2.

Let us now discuss the $\mathrm{nH}$ analysis. 217 days of data taking corresponding to the Daya Bay time period when only 6 ADs were functioning have been used in this analysis. Since $n+p \rightarrow$ ${ }^{2} \mathrm{D}+\gamma$ reaction releases smaller energy $(2.2 \mathrm{MeV}$ ) than $\mathrm{Gd}$ excitations (about $8 \mathrm{MeV}$ ) there are more accidentals due to the lower delayed energy threshold. This is one example of generally somewhat different systematics relative to $\mathrm{nGd}$ analysis.

As a result of $\mathrm{nH}$ analysis the far detectors also observe a deficit in the event rate compared to the expectations based on near detectors measurements. Within the three-neutrino oscillation framework it allows us to measure $\sin ^{2} 2 \theta_{13}=0.083 \pm 0.018$ in good agreement with $\mathrm{nGd}$ analysis. While $\mathrm{nH}$ spectral analysis is in progress one could observe that the spectral distortion is consistent with oscillations as can be seen from the right panel of Fig. 2.

\subsection{Absolute reactor neutrino flux measurement}

The large reactor $\bar{v}_{e}$ sample collected at Daya Bay allows for a precise measurement of the absolute reactor antineutrino flux. The analysis uses the complete 217-day data set of the 6-AD period. A total 

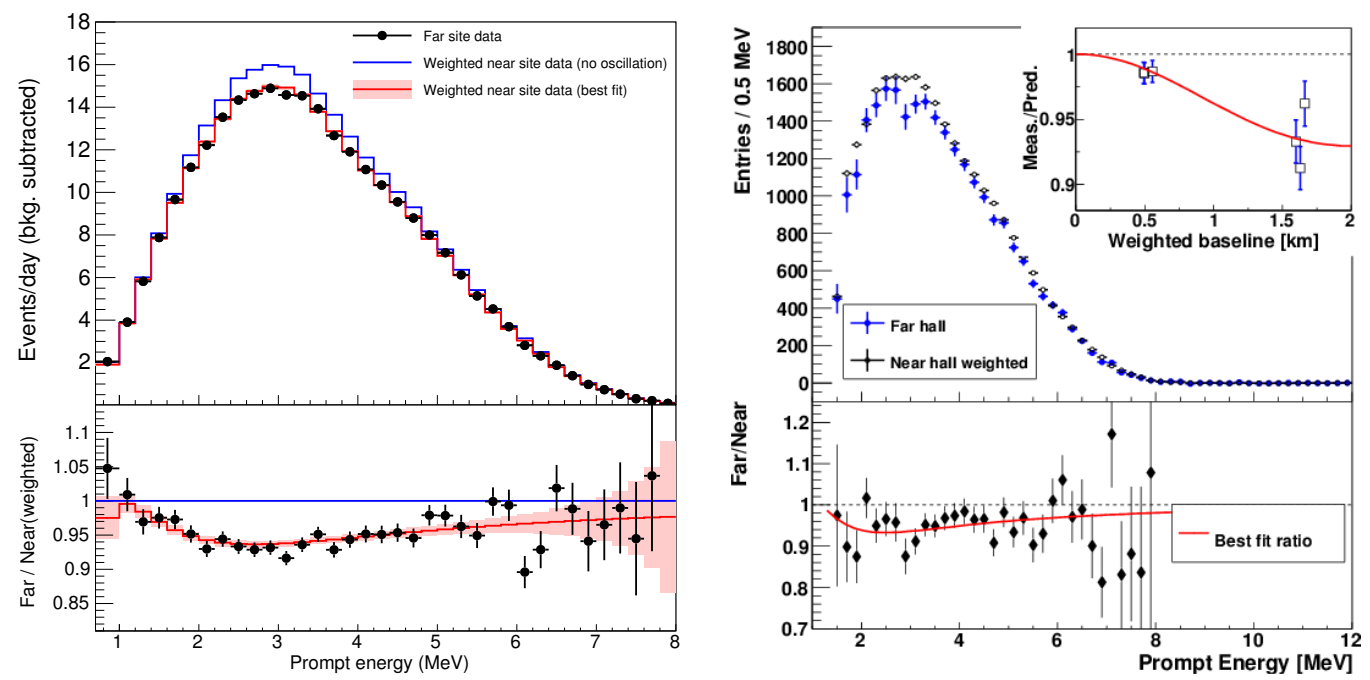

Figure 2. Left panel: nGd analysis. The upper panel shows the background substracted prompt positron spectra (black points) measured in the far experimental hall. The blue line shows the expectations based on the near site data assuming no oscillation. The red line represents the expectations based on the near site data assuming best fit oscillation. The band corresponds to the uncertainty in the prediction. In the lower panel the black points represent the ratio of the background-subtracted data divided by the predicted no-oscillation spectra. The error bars represent the statistical uncertainty only. The red curve in each lower panel represents the ratio of the best-fit to no-oscillations spectra. The change in slope of the red curve in the lowest energy bin is due to the effect of energy loss in the acrylic. Right panel: $\mathrm{nH}$ analysis. The detected energy spectrum of the prompt events of the far hall ADs (blue) and near hall ADs (open circle) weighted according to baseline. The far-to-near ratio (solid dot) with best fit $\theta_{13}$ value is shown in the lower plot. In the inset is the ratio of the measured to the predicted rates in each $\mathrm{AD}$ vs baseline, in which the AD4 (AD6) baseline was shifted relative to that of AD 5 by $30(-30)$ $\mathrm{m}$ for visual clarity.

of $300 \mathrm{k}(40 \mathrm{k})$ candidates are detected at the near (far) halls. Fig. 3 shows the measured reactor $\bar{v}_{e}$ event rate at each $\mathrm{AD}$ after correcting for the $\bar{v}_{e}$ survival probability, re-expressed as $Y_{0}\left(\mathrm{~cm}^{2} \mathrm{GW}^{-1}\right.$ day $\left.^{-1}\right)$ and $\sigma_{f}\left(\mathrm{~cm}^{2}\right.$ fission $\left.{ }^{-1}\right)$. The measurement among ADs is consistent within statistical fluctuations after correcting for the difference in the effective fission fractions. The uncertainty (2.3\%) of the measurement is dominated by the uncertainty in detection efficiency $(2.1 \%)$, which is correlated among all ADs. The measurement yields an average $Y_{0}=1.553 \times 10^{-18} \mathrm{~cm}^{2} \mathrm{GW}^{-1} \mathrm{day}^{-1}$ and $\sigma_{f}=5.934 \times 10^{-43} \mathrm{~cm}^{2}$ fission ${ }^{-1}$, with the average fission fractions ${ }^{235} \mathrm{U}:{ }^{238} \mathrm{U}:{ }^{239} \mathrm{Pu}:{ }^{241} \mathrm{Pu}=$ $0.586: 0.076: 0.288: 0.050$. Three theoretical model predictions are shown in Fig. 3 as a reference. The Huber [32] and ILL [33, 34] models predict the $\bar{v}_{e}$ spectra for ${ }^{235} \mathrm{U},{ }^{239} \mathrm{Pu}$ and ${ }^{241} \mathrm{Pu}$, while the Mueller [35] and Vogel [36] models predict for ${ }^{238} \mathrm{U}$. The uncertainty in the model predictions is estimated by authors to be $\simeq 2.7 \%$. This estimate might be somewhat optimistic as follows from [37] which suggests the corresponding uncertainty to be not less than $4 \%$. The ratio $(\mathrm{R})$ of the Daya Bay measurement to the Huber+Muller model prediction is $R=0.947 \pm 0.022$, while $R=0.992 \pm 0.023$ when compared to the ILL+Vogel model prediction.

The Daya Bay result is compared to the 21 past reactor neutrino flux measurements as shown in Fig. 4 according to Refs. [38, 39]. As the common reference model for all experiments in Fig. 4 the Huber+Mueller model is used assuming the neutron lifetime value to be $880.1 \mathrm{~s}$ [40]. The 


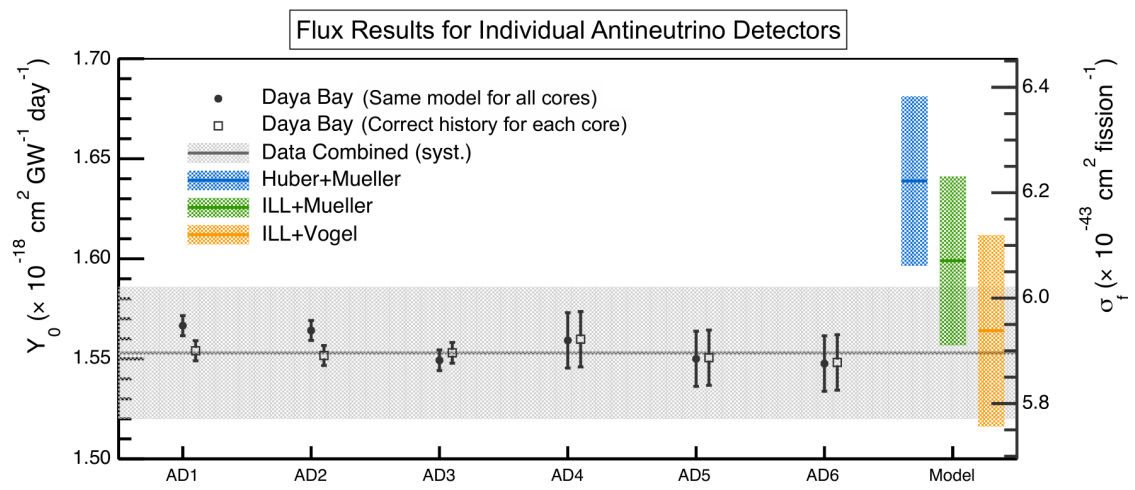

Figure 3. The measured reactor $\bar{v}_{e}$ event rate at each $\mathrm{AD}$ after correcting for the $\bar{v}_{e}$ survival probability, reexpressed as $Y_{0}\left(\mathrm{~cm}^{2} \mathrm{GW}^{-1} \mathrm{day}^{-1}\right)$ and $\sigma_{f}\left(\mathrm{~cm}^{2}\right.$ fission $\left.{ }^{-1}\right)$. The solid and open circles show the data without and with correction for the difference in the effective fission fractions observed by each AD. The uncertainty of the measurement is shown as the gray band. Three theoretical model predictions are shown as a reference.

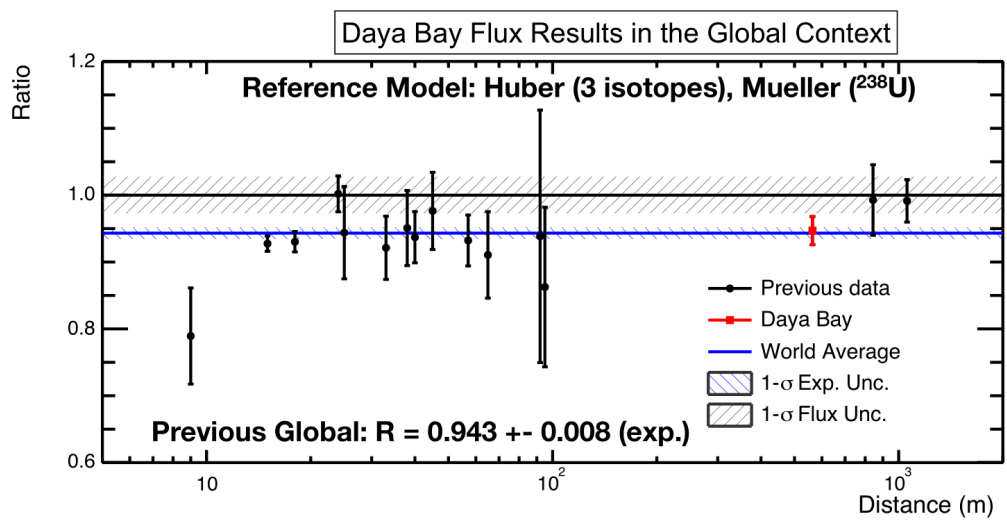

Figure 4. The reactor $\bar{v}_{e}$ interaction rate of the 21 previous short-baseline experiments $[38,39]$ as a function of the distance from the reactor, normalized to the Huber+Mueller model prediction [32, 35]. Experiments at the same baseline are combined together for clarity. The Daya Bay experiment is placed at the effective baseline of $573 \mathrm{~m}$. The rate is corrected by the $\bar{v}_{e}$ survival probability at the distance of each experiment, assuming standard three-neutrino oscillation. The horizontal bar (blue) represents the global average and its $1 \sigma$ uncertainty. The $2.7 \%$ reactor flux uncertainty is shown as a band around unity.

$\bar{v}_{e}$ survival probability is calculated with $\sin ^{2} 2 \theta_{13}=0.089 \pm 0.009$ determined from the rate-only analysis [27]. The global average of the 21 past measurements with respect to the Huber+Mueller model prediction is determined to be $R=0.943 \pm 0.008$ (experimental uncertainty), which is consistent with $R=0.947 \pm 0.022$ from the Daya Bay measurement. 


\subsection{Energy spectrum measurement}

A preliminary comparison of the measured prompt energy spectrum to the expectations based on Huber+Muller model prediction [32, 35] is displayed in Fig. 5.

One can observe a significant mismatch of the spectra in the energy region $4-6 \mathrm{MeV}$ where the local significance of the discrepancy reaches the level of $4 \sigma$. This excess is observed also by RENO [41] and Double Chooz [42] reactor experiments. The excess is unlikely to be caused by unaccounted for detector effects or additional background. It matches all characteristics of IBD events. It correlates to the reactor power and apart of that is time independent. First-principle calculations of fission and $\beta$ decay processes predict similar excess [43] where the authors conclude "The presence of this bump in both the calculated electron and antineutrino spectra suggests that the discrepancy may not be due to systematics of the $\beta^{-}$conversion method, but instead may be an artifact of the original $\beta^{-}$measurements". Today the origin of this descrepancy is an open question.

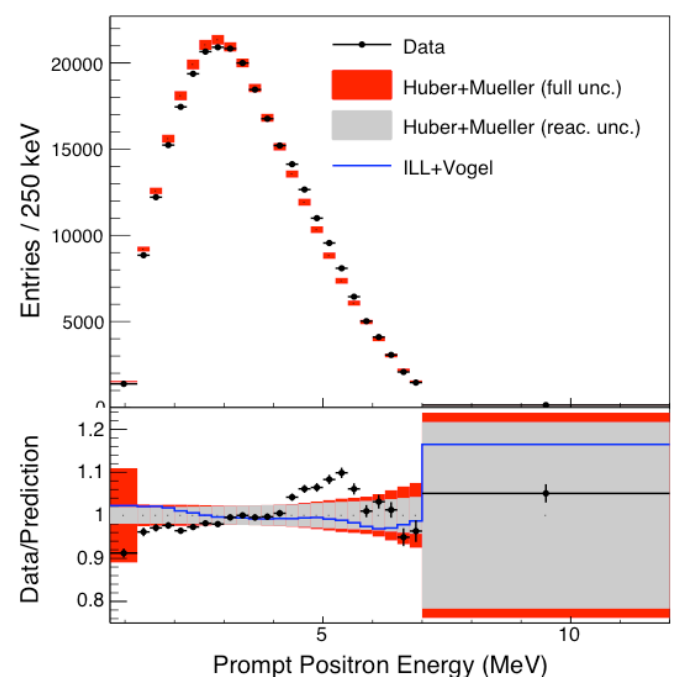

Figure 5. Upper panel: the measured prompt energy spectrum by Daya Bay experiment compared to the expectations based on Huber+Muller model prediction $[32,35]$. Bottom panel: data/prediction ratio. The shadowed area respresents the theory model estimation of the uncertainty.

\subsection{Light sterile neutrino search}

The Daya Bay experiment performed a search for a possible sterile neutrino. What is the sterile neutrino? It is a quantum state defined as a coherent ("flavor") mixture of massive states $v_{1}, v_{2}, v_{3}, v_{4}$, etc which does not interact with $W^{ \pm}, Z$. However each of massive $v_{i}$ does interact with gauge bosons. The $4 \times 4$ (in a minimal extension of the Standard Model) unitary mixing matrix is organized in such a way that four massive neutrinos contribute as just three states to the widths of $W^{ \pm}, Z$. However it does not mean that fourth (or more) massive neutrino remains invisible. If an initially produced flavour state $\left(v_{e}, v_{\mu}, v_{\tau}\right)$ evolves with time it might appear as sterile state thus making additional deficit of the detected events. In Daya Bay the sterile neutrino could cause additional spectral distortion betweens the ADs thanks to multiple baselines $(350 \mathrm{~m}, 500 \mathrm{~m}$ and $1600 \mathrm{~m})$ as shown in the left panel of Fig. 6 . 

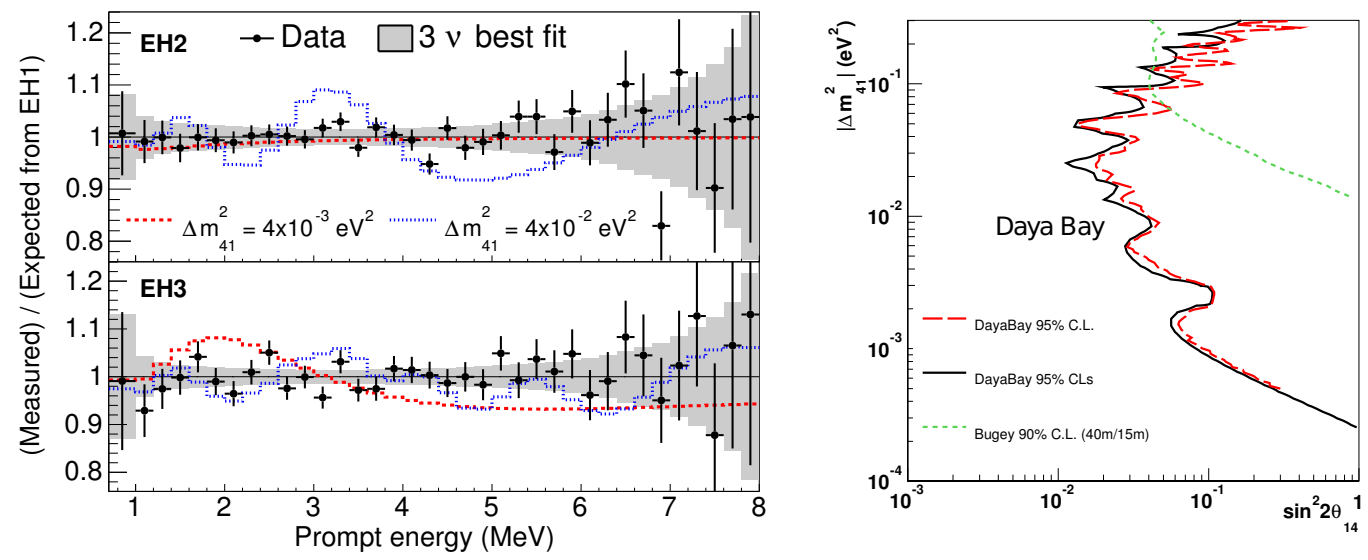

Figure 6. Left panel: Prompt energy spectra observed at EH2 (top) and EH3 (bottom), divided by the prediction from the EH1 spectrum with the three-neutrino best fit oscillation parameters from the previous Daya Bay analysis [27]. The gray band represents the uncertainty of three-neutrino oscillation prediction, which includes the statistical uncertainty of the EH1 data and all the systematic uncertainties. Predictions with $\sin ^{2} 2 \theta_{14}=0.1$ and two representative $\left|\Delta m_{41}^{2}\right|$ values are also shown as the dotted and dashed curves. Right panel: The exclusion contours for the neutrino oscillation parameters $\sin ^{2} 2 \theta_{14}$ and $\left|\Delta m_{41}^{2}\right|$. Normal mass hierarchy is assumed for both $\Delta m_{31}^{2}$ and $\Delta m_{41}^{2}$. The red long-dashed curve represents the 95\% C.L. exclusion contour with Feldman-Cousins method [44]. The black solid curve represents the $95 \% \mathrm{CL}_{\mathrm{s}}$ exclusion contour [47]. The parameter-space to the right side of the contours are excluded. For comparison, Bugey's [48] 90\% C.L. limit on $\bar{v}_{e}$ disappearance is also shown as the green dashed curve.

The analysis uses the complete 217-day data set of the 6-AD period. The relative spectral distortion due to the disappearance of $\bar{v}_{e}$ is found to be consistent with that of the three-flavor oscillation model. The exclusion contours for $\sin ^{2} 2 \theta_{14}$ and $\left|\Delta m_{41}^{2}\right|$ displayed in the right panel of Fig. 6 are determined using both the Feldman-Cousins method [44] and the CLs method [45]. The derived limits cover the $10^{-3} \mathrm{eV}^{2}<\left|\Delta m_{41}^{2}\right|<0.1 \mathrm{eV}^{2}$ region, which was previously largely unexplored. Details of the sterile neutrino analysis can be found in [46].

\section{Summary}

The Daya Bay experiment uses the relative measurement of the $\bar{v}_{e}$ rate and spectrum between near and far detectors to precisely measure the oscillation parameters $\sin ^{2} 2 \theta_{13}$ and $\left|\Delta m_{\mathrm{ee}}^{2}\right|$. The Daya Bay experiments takes the data in the final 8-AD configuration since summer 2012 when two new ADs were installed. With 621 days of data, Daya Bay has measured $\sin ^{2} 2 \theta_{13}=0.084 \pm 0.005$ and $\left|\Delta m_{\mathrm{ee}}^{2}\right|=2.44_{-0.11}^{+0.10} \times 10^{-3} \mathrm{eV}^{2}$. This is the most precise measurement of $\sin ^{2} 2 \theta_{13}$ to date. The precision measurement of $\theta_{13}$ opens the door for future experiments to study neutrino mass hierarchy and leptonic CP violation. The $\left|\Delta m_{\mathrm{ee}}^{2}\right|$ measurement is in agreement with $\left|\Delta m_{\mu \mu}^{2}\right|$ measurements by the muon neutrino disappearance experiments. The precisions of both $\left|\Delta m_{\mathrm{ee}}^{2}\right|$ and $\left|\Delta m_{\mu \mu}^{2}\right|$ measurements are comparable today. By the end of 2017 Daya Bay expects to measure both $\sin ^{2} 2 \theta_{13}$ and $\left|\Delta m_{\mathrm{ee}}^{2}\right|$ with precisions better than $3 \%$.

Several other analyses have been also performed. $\theta_{13}$ angle has been measured in $\mathrm{nH}$ analysis yielding $\sin ^{2} 2 \theta_{13}=0.083 \pm 0.018$. The absolute reactor antineutrino flux measurement has yielded 
results consistent with previous short-baseline reactor neutrino experiments thus confirming the "Reactor Antineutrino Anomaly" first introduced in Ref. [38]. However it is still an open question if the anomaly is due to sterile neutrinos or due to uncertainties in the model calculations of reactor antineutrino fluxes. Therefore, an analysis based mostly on the energy shape information and exploiting multiple baselines of Daya Bay experiment has been performed searching for a possible signal of sterile neutrino in the observed energy spectra. Such a signal has not been observed which allows us to set stringent limits in the $10^{-3} \mathrm{eV}^{2}<\left|\Delta m_{41}^{2}\right|<0.1 \mathrm{eV}^{2}$ region. Finally, preliminary results on energy spectrum of reactor antineutrino show generally a good agreement with expectations [32, 35] except the energy interval $4-6 \mathrm{MeV}$ with where a significant mismatch has been observed.

\section{ACKNOWLEDGMENTS}

Daya Bay is supported in part by the Ministry of Science and Technology of China, the U.S. Department of Energy, the Chinese Academy of Sciences, the National Natural Science Foundation of China, the Guangdong provincial government, the Shenzhen municipal government, the China General Nuclear Power Group, Key Laboratory of Particle and Radiation Imaging (Tsinghua University), the Ministry of Education, Key Laboratory of Particle Physics and Particle Irradiation (Shandong University), the Ministry of Education, Shanghai Laboratory for Particle Physics and Cosmology, the Research Grants Council of the Hong Kong Special Administrative Region of China, the University Development Fund of The University of Hong Kong, the MOE program for Research of Excellence at National Taiwan University, National Chiao-Tung University, and NSC fund support from Taiwan, the U.S. National Science Foundation, the Alfred P. Sloan Foundation, the Ministry of Education, Youth, and Sports of the Czech Republic, the Joint Institute of Nuclear Research in Dubna, Russia, the CNFC-RFBR joint research program, the National Commission of Scientific and Technological Research of Chile, and the Tsinghua University Initiative Scientific Research Program. We acknowledge Yellow River Engineering Consulting Co., Ltd., and China Railway 15th Bureau Group Co., Ltd., for building the underground laboratory. We are grateful for the ongoing cooperation from the China General Nuclear Power Group and China Light and Power Company.

I would like also to warmly thank all my collegues from the Daya Bay Collaboration and especially Maxim Gonchar, Sören Jetter, Jiajie Ling, Logan Lebanowski, Pedro Ochoa, Xin Qian, Wei Wang, Zhe Wang, Chao Zhang, Weili Zhong for useful discussions and providing me with supplemental material for this manuscript.

\section{References}

[1] B. Pontecorvo, Sov. Phys. JETP 6, 429 (1957) [Zh. Eksp. Teor. Fiz. 33, 549 (1957)].

[2] B. Pontecorvo, Sov. Phys. JETP 26, 984 (1968) [Zh. Eksp. Teor. Fiz. 53, 1717 (1967)].

[3] M. Beuthe, Phys. Rev. D 66, 013003 (2002) [hep-ph/0202068].

[4] C. Y. Cardall, Phys. Rev. D 61, 073006 (2000) [hep-ph/9909332].

[5] P. Stockinger, Pramana 54, 203 (2000).

[6] W. Grimus, S. Mohanty and P. Stockinger, Phys. Rev. D 61, 033001 (2000) [hep-ph/9904285].

[7] W. Grimus, S. Mohanty and P. Stockinger, hep-ph/9909341.

[8] W. Grimus, S. Mohanty and P. Stockinger, In *Cape Town 1999, Weak interactions and neutrinos* 355-359 [hep-ph/9904340].

[9] E. K. Akhmedov, JHEP 0709, 116 (2007) [arXiv:0706.1216 [hep-ph]].

[10] E. K. Akhmedov and A. Wilhelm, JHEP 1301, 165 (2013) [arXiv:1205.6231 [hep-ph]]. 
[11] E. K. Akhmedov and J. Kopp, JHEP 1004, 008 (2010) [Erratum-ibid. 1310, 052 (2013)] [arXiv:1001.4815 [hep-ph]].

[12] D. V. Naumov and V. A. Naumov, J. Phys. G 37, 105014 (2010) [arXiv:1008.0306 [hep-ph]].

[13] D. V. Naumov and V. A. Naumov, Russ. Phys. J. 53, 549 (2010) [Izv. Vuz. Fiz. 6, 5 (2010)].

[14] S. Abe et al. [KamLAND Collaboration], Phys. Rev. Lett. 100, 221803 (2008) [arXiv:0801.4589 [hep-ex]].

[15] P. Adamson et al. [MINOS Collaboration], Phys. Rev. Lett. 110, no. 17, 171801 (2013) [arXiv:1301.4581 [hep-ex]].

[16] Y. Ashie et al. [Super-Kamiokande Collaboration], Phys. Rev. Lett. 93, 101801 (2004) [hepex/0404034].

[17] H. Nunokawa, S. J. Parke and R. Zukanovich Funchal, Phys. Rev. D 72, 013009 (2005) [hep$\mathrm{ph} / 0503283]$.

[18] M. Apollonio et al. [CHOOZ Collaboration], Eur. Phys. J. C 27, 331 (2003) [hep-ex/0301017].

[19] P. Adamson et al. [MINOS Collaboration], Phys. Rev. Lett. 107, 181802 (2011) [arXiv:1108.0015 [hep-ex]].

[20] K. Abe et al. [T2K Collaboration], Phys. Rev. Lett. 107, 041801 (2011) [arXiv:1106.2822 [hepex]].

[21] Y. Abe et al. [DOUBLE-CHOOZ Collaboration], Phys. Rev. Lett. 108, 131801 (2012) [arXiv:1112.6353 [hep-ex]].

[22] G.L. Fogli et al., Phys. Rev. D 84, 053007 (2011)

[23] F. P. An et al. [DAYA-BAY Collaboration], Phys. Rev. Lett. 108, 171803 (2012) [arXiv:1203.1669 [hep-ex]].

[24] J. K. Ahn et al. [RENO Collaboration], Phys. Rev. Lett. 108, 191802 (2012) [arXiv:1204.0626 [hep-ex]].

[25] Chao Zhang, "Results from Daya Bay", NEUTRINO 2014

[26] Weili ZHONG, "Measurement of Reactor Antineutrino Flux and Spectrum at Daya Bay", ICHEP 2014

[27] F. P. An et al. [Daya Bay Collaboration], Phys. Rev. Lett. 112, 061801 (2014) [arXiv:1310.6732 [hep-ex]].

[28] F. P. An et al. [Daya Bay Collaboration], Phys. Rev. D 90, no. 7, 071101 (2014) [arXiv:1406.6468 [hep-ex]].

[29] F. P. An et al. [Daya Bay Collaboration], Nucl. Instrum. Meth. A 685, 78 (2012) [arXiv:1202.6181 [physics.ins-det]].

[30] X. Guo et al. [Daya-Bay Collaboration], hep-ex/0701029.

[31] F. P. An et al. [Daya Bay Collaboration], Chin. Phys. C 37, 011001 (2013) [arXiv:1210.6327 [hep-ex]].

[32] P. Huber, Phys. Rev. C 84, 024617 (2011) [Erratum-ibid. C 85, 029901 (2012)] [arXiv:1106.0687 [hep-ph]].

[33] K. Schreckenbach, G. Colvin, W. Gelletly and F. Von Feilitzsch, Phys. Lett. B 160, 325 (1985).

[34] A. A. Hahn, K. Schreckenbach, G. Colvin, B. Krusche, W. Gelletly and F. Von Feilitzsch, Phys. Lett. B 218, 365 (1989).

[35] T. A. Mueller, D. Lhuillier, M. Fallot, A. Letourneau, S. Cormon, M. Fechner, L. Giot and T. Lasserre et al., Phys. Rev. C 83, 054615 (2011) [arXiv:1101.2663 [hep-ex]].

[36] P. Vogel, G. K. Schenter, F. M. Mann and R. E. Schenter, Phys. Rev. C 24, 1543 (1981). 
[37] A. C. Hayes, J. L. Friar, G. T. Garvey, G. Jungman and G. Jonkmans, Phys. Rev. Lett. 112, 202501 (2014) [arXiv:1309.4146 [nucl-th]].

[38] G. Mention, M. Fechner, T. Lasserre, T. A. Mueller, D. Lhuillier, M. Cribier and A. Letourneau, Phys. Rev. D 83, 073006 (2011) [arXiv:1101.2755 [hep-ex]].

[39] C. Zhang, X. Qian and P. Vogel, Phys. Rev. D 87, no. 7, 073018 (2013) [arXiv:1303.0900 [nuclex]].

[40] J. Beringer et al. [Particle Data Group Collaboration], Phys. Rev. D 86, 010001 (2012).

[41] S. H. Seo [for the RENO Collaboration], arXiv:1410.7987 [hep-ex].

[42] J. I. Crespo-Anadon [Double Chooz Collaboration], arXiv:1412.3698 [hep-ex].

[43] D. A. Dwyer and T. J. Langford, arXiv:1407.1281 [nucl-ex].

[44] G. J. Feldman and R. D. Cousins, Phys. Rev. D 57, 3873 (1998) [physics/9711021 [physics.dataan]].

[45] X. Qian, A. Tan, J. J. Ling, Y. Nakajima and C. Zhang, arXiv:1407.5052 [hep-ex].

[46] F. P. An et al. [DAYA-BAY Collaboration], Phys. Rev. Lett. 113, 141802 (2014) [arXiv:1407.7259 [hep-ex]].

[47] A. L. Read, J. Phys. G 28, 2693 (2002).

[48] Y. Declais, J. Favier, A. Metref, H. Pessard, B. Achkar, M. Avenier, G. Bagieu and R. Brissot et al., Nucl. Phys. B 434, 503 (1995). 